\title{
Fracture of Cylindrical Shells Containing a Circumferential Crack
}

\section{KUMOSA* and D. HULL}

Department of Materials Science and Metallurgy, University of Cambridge, Pembroke Street, Cambridge CB2 3QX, UK

ABSTRACT

A finite element analysis is developed to determine all possible fracture modes at the tip of a circumferential through-crack in cylindrical shells. Two different types of loading are used which assume either the application of external torque or axial tension. Some numerical results are also presented for the shells subjected to combined tension and torsion. Fracture properties of the shells are examined as a function of crack length. It is shown that for very short cracks in thin-walled cylindrical shells the stress intensity factors for the individual cases of tension or torsion can be determined from the shallow-shell theory. For long cracks however, disagreement between the finite element data and the shallow-shell theory results is very pronounced. It is observed that combined loading produces extremely complicated displacements at the crack tip which are particularly visible for long cracks.

KEYWORDS

Cylindrical Shell; Circumferential Crack; FEM; Stress Intensity Factors: Tension; Torsion.

INTRODUCTION

The derivation of stress intensity factors for circumferentially cracked cylindrical shells is of great importance in design practice. It is essential in safety analysis of structures in such fields as pressure vessels, off-shore production and piping, aircraft and aerospace engineering to consider the behaviour of stresses around the cracks for the prediction of the safe life and the critical crack length. The loading conditions for most crack cases are either axial tension, bending or torsion, or a
combination of all loading modes.

The present paper describes the finite element calculations of thin-walled cylinders with circumferential through-cracks, subjected to either uniform tensile loading or axial torsion. A combination of both of the loading modes is also considered. The problems have been previously examined by classical methods using shallow-shell theory and perturbation techniques in tension (Folias, 1967; Erdogan and Ratwani, 1970; Duncan - Fama and Sanders, 1972; Delale and Erdogan, 1979; Murthy et a1, 1974), and in torsion

(Erdogan and Ratwani, 1972; Lakshminarayana and Murthy, 1976). Shallow-shel

*On leave from Wroclaw Technical University, Poland. 
theory and perturbation techniques give only an approximate solution valid for cracks of small lengths. Due to the mathematical complexities of all stress intensity factors for through-cracks in cylindrical shells, the functional dependence of these factors upon shell geometry, crack length, applied load and stiffness coefficients of a shell can be established only through such numerical methods as PEM.

\section{EVALUATION OF STRESS INTEISITY FACTORS}

The shells were stressed such that in the absence of the crack, a uniaxial tensile or in-plane shear stress would be produced which are uniform along the crack side and uniform through the thickness. The presence of the crack changes the stress distribution and causes the bending stresses which vary linearly through the thickness of the shell. If the thickness to radius ratio $(t / R)$ and the crack length a are sufficiently small, then the general solution of the stress intensity factors in the mode I or II loading may be obtained by approximately superimposing the results of membrane and bending solution with

$$
\mathrm{K}_{\mathrm{I}, \mathrm{II}} / \mathrm{K}_{\mathrm{o}}^{(\mathrm{I}, \mathrm{II})}=\mathrm{G}_{\mathrm{m}}{ }^{(\mathrm{I}, \mathrm{II})}+\mathrm{G}_{\mathrm{b}}^{\mathrm{I}, \mathrm{II})}(2 \mathrm{y} / \mathrm{t})
$$

where

$G(I, I I)$ and $G(I, I I)$ are non-dimensional membrane and bending stress components in bension (I) and in torsion (II). The coordinance $y$ is equal to $t t / 2$ on the outer surface and $-5 / 2$ on the inner surface of the shell. The stress intensity factors $\mathrm{K}(\mathrm{I}, \mathrm{I})$ can be evaluated for a cracked flat plate subjected to the same loading as the circumferential crack in the cylinder and are given by

$$
\begin{aligned}
& K_{0}{ }^{(I)}=\sigma_{m} \sqrt{\pi a}((2 b / \pi a) \tan (\pi a / 2 b))^{1 / 2} \\
& K_{0}(I I)=\tau_{m} \sqrt{\pi a}\left(T / 2 \pi R^{2} t\right) \sqrt{\pi a}
\end{aligned}
$$

where

$$
\begin{aligned}
\sigma_{m} & =\text { membrane tensile stress } \\
2 \mathrm{~b} & =\text { cylinder circumference } \\
\tau_{\mathrm{m}} & =\text { membrane shear stress } \\
\mathrm{T} & =\text { torque }
\end{aligned}
$$

The stress intensity components $G_{m}$ and $G$ were determined from the finite element analysis knowing the numerical displacements near the crack tip. The stress intensity factors were evaluated from the following equation. (Shih et al., 1976)

$$
\mathrm{K}_{\mathrm{I}, \mathrm{II}}=\frac{2 \sqrt{2 \pi}}{\mathrm{K}+1} \frac{\mu}{\sqrt{1}}\left(4 \mathrm{u}_{B}-u_{\mathrm{C}}\right)
$$

where $\mathrm{u}_{B}$ and $\mathrm{u}_{C}$ are the displacements (opening in tension and edge sliding in
shear) calculated at the quarter point node (B) and the edge node (C) of the crack tip elements respectively.
1 - is the length of the singular crack element along the crack face $\mu$ - is the shear modulus

$K=3-4 \nu-$ for plane strain and $K=(3-\nu) /(1+\nu)$ for plane stress.

The stress intensity factors were calculated for both plane stress and plane strain conditions and their average values were taken into consideration.

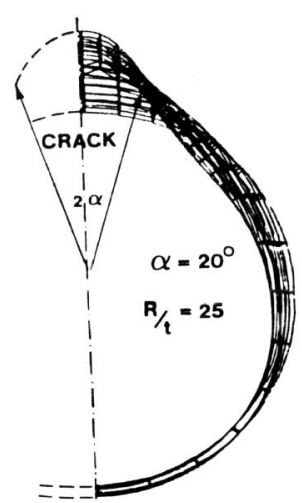

Fig.1. Displaced finite element mesh view along the cylinder axis.

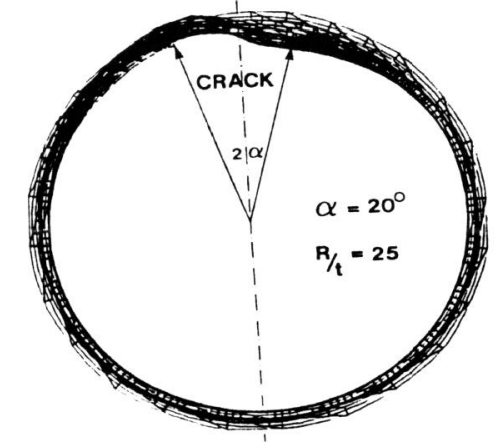

Fig. 2. Displaced finite element in torsion; view along
NUMERICAL RESULTS AND DISCUSSION

The deformation of thin-walled cylindrical shells is highly dependent upon the specimen geometry, elastic properties and loading conditions. Figure 1 and 2 represent the displaced shapes of two finite element meshes containing circumferential cracks with the half angular crack length $\alpha=20^{\circ}$ and $R / t$ $25(\mathrm{R}=25 \mathrm{~mm}, t=1 \mathrm{~mm})$ loaded $1 \mathrm{n}$ tens apparent that the mesh deformation is significantion (bulging effect) around There is a symmetric out-of-plane shear deforma Kumosa and Hu11, 1988a, the crack in tension (Delale and Erdogan, 1Tion. (Kumosa and Hull, $1988 \mathrm{c})$ and an antisymmetric shear deforintion $1988 \mathrm{~b}, 1988 \mathrm{c}$ ) with two crack faces crossing each for membrane and bending Figures 3 and 4 show the finiter under stress intensity ratios $m$ and $G_{b}$ respective

$$
\lambda_{2}=\left[12\left(1-v^{2}\right)\right]^{1 / 4} \mathrm{a} /(\mathrm{Rt})^{1 / 2}
$$

for constant ratio $R / t=25$. The finite element data (points on continuous lines) is compared with the shallow-shell theory results (Delale and geometries and $v=0.33$. For the $\lambda_{2}$ smaller than 3.0 (short cracks) the 
membrane stress intensity factors in tension and torsion are in good agreement with the values obtained from the shallow-shell theory.

Increasing the half angular crack length over approximately $23^{\circ}$ shows a significant increase in the finite element $G_{m}$ values.

Similar to $G_{m}$, the shallow-shell theory results $G_{p}$ (Fig.4) are different for large $\lambda_{2}$ to those obtained from the finite element analysis of a shell in tension. In torsion, however, the bending component $G$ determined from the shallow-shell theory is of the order $\pm 4 \times 10^{-}$(Erdogan and Ratwani, 1972)

significantly greater. The reason for this difference is that the bending

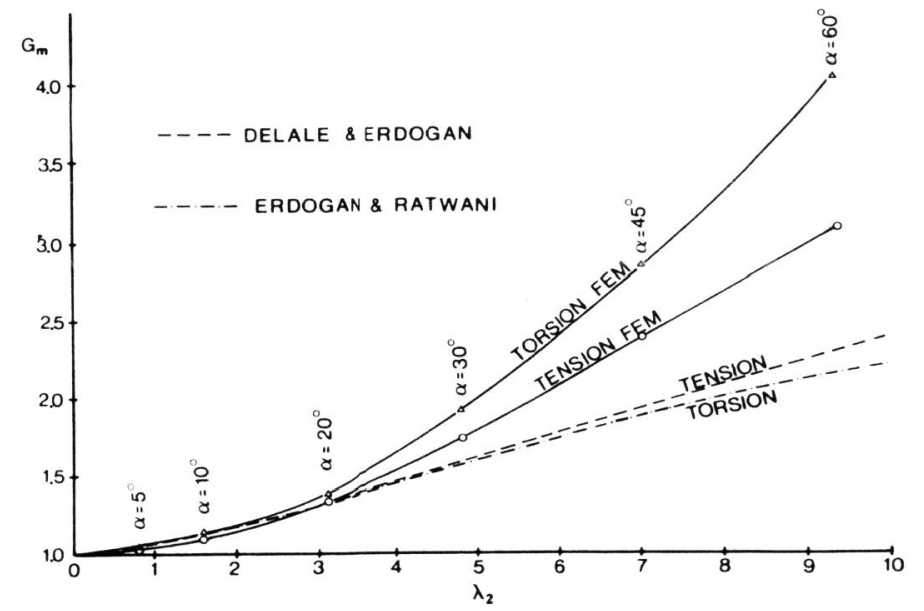

Fig. 3 Membrane stress intensity components $G_{m}$ for tubes with $R / t=25$ and a ranging from 5 to $60^{\circ}$.

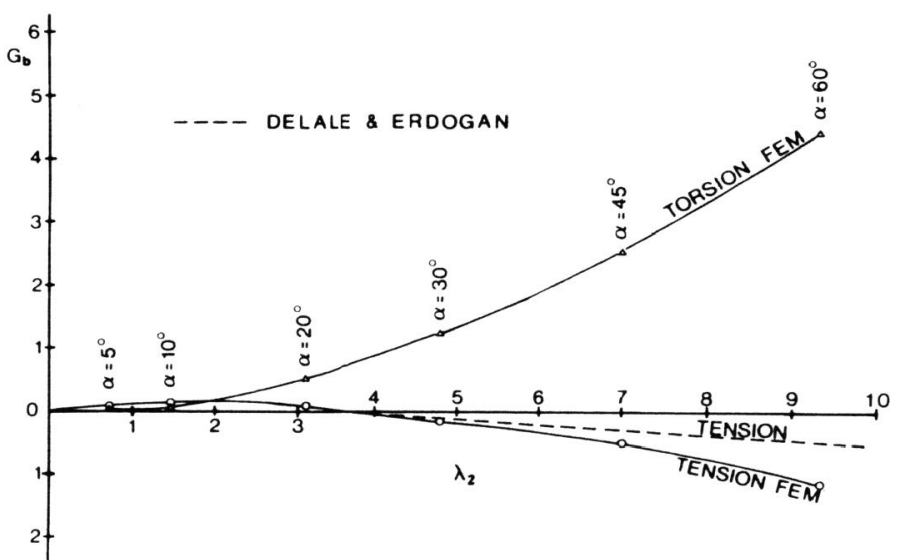

Fig. 4 Bending stress intensity components $G_{b}$ for tubes with $\mathrm{R} / \mathrm{t}=25$ and $\alpha$ ranging from $5^{\circ}$ to $60^{\circ \mathrm{b}}$ component is very sensitive to the transverse shear effect which was not taken into consideration in the shallow-shell theory.

The out-of-plane symmetric deformation has been analysed by Delale and Erdogan, (1979) for an isotropic cylinder with $\lambda_{2}=3$ and $a / t=5$ for $v$ 0.33 . For comparison the normalised $\bar{\omega}$ components of the crack surface displacements obtained from the finite element analysis and by Delale and Erdogan (1979) are presented in Fig.5. The agreement between them is quite close. The out-of-plane deformation affects the stress intensity factors $G_{m}$ and $G_{b}$ without changing, however, the type of loading at the crack tip.

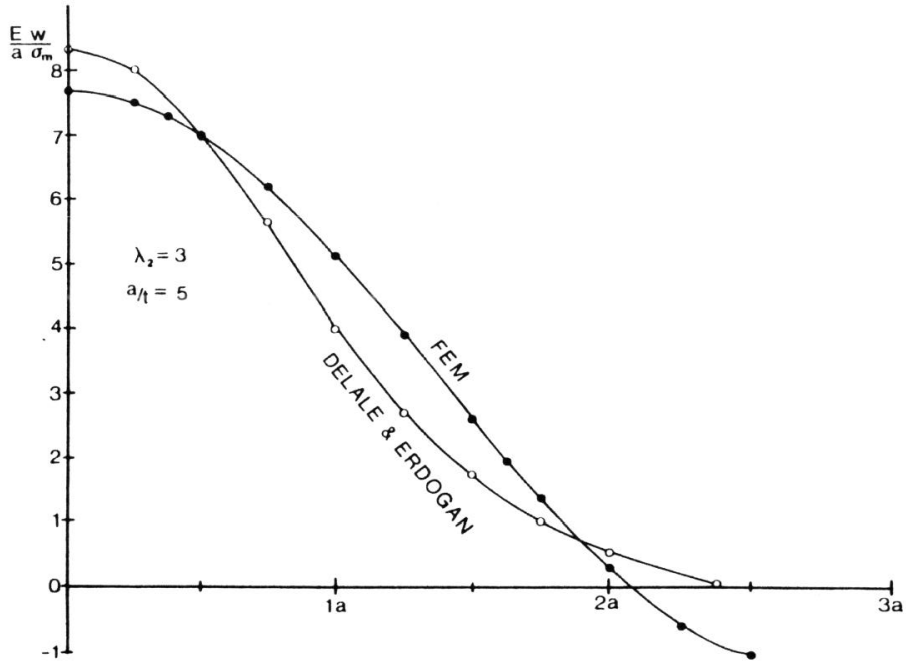

Fig.5. Out-of-plane normalised components $\bar{\omega}$ of the crack surface displacements for a cylinder in tension with $\lambda_{2}=3$ and $a / t=5$.

It has been reported (Kumosa and Hu11, 1988b) that the out-of-p1ane antisymmetric shear deformation near the crack produces very complicated displacements at the crack tip for a shell subjected to torsion. For short crack tip whearas for longer cracks mixed mode loading occurs with crack opening and tearing displacmes. The displacent at two crack faces obtaind are shown in Fig.6. $s$ rees to the displas outer, middle and inner surface $S$ indicates the displacmenets of nodes at a distance of on to the displacements of corner nodes at a distance of one, two $1,2,4$ refer four elents from the crack tip along the crak face. It has been shown that for short cracks and hick allod and $U$ (crack opening and tearing displaling her lor thints) are fracture occurs.

Extending the analysis to the case of combined tension and torsion the entire cylinder was modelled. As an example, the deformation of the finite 
element mesh with $R / t=25$ and $\alpha=20^{\circ}$ is shown in Fig.7. The combined loading produces different mixed mode loading modes at the crack tip for different crack lengths. For very short cracks the membrane and bending

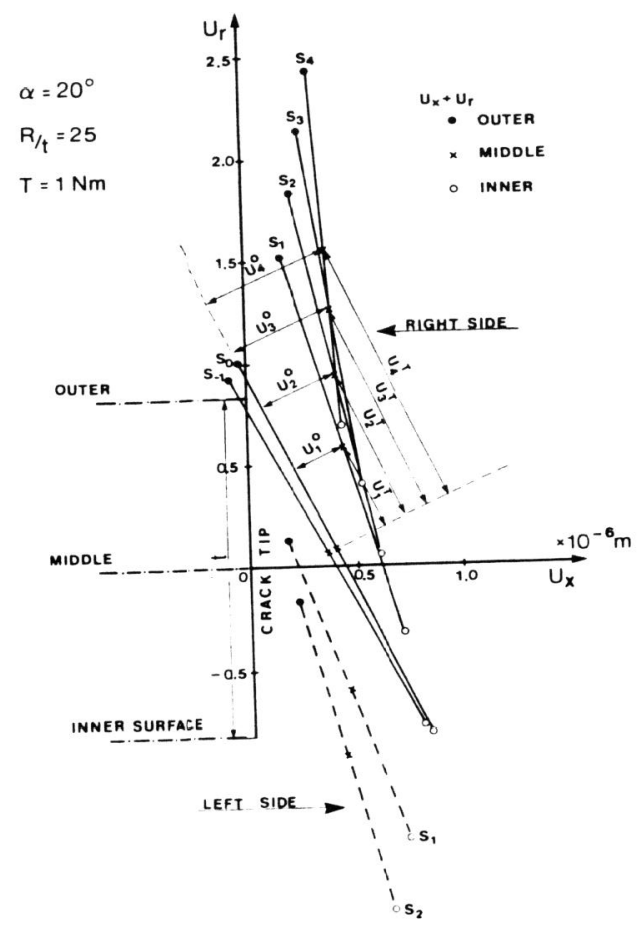

Fig. 6 Axial $U_{\text {and radial }} U_{r}$ displacements of two crack faces and evałuated crack opeping displacements and crack tearing displacements

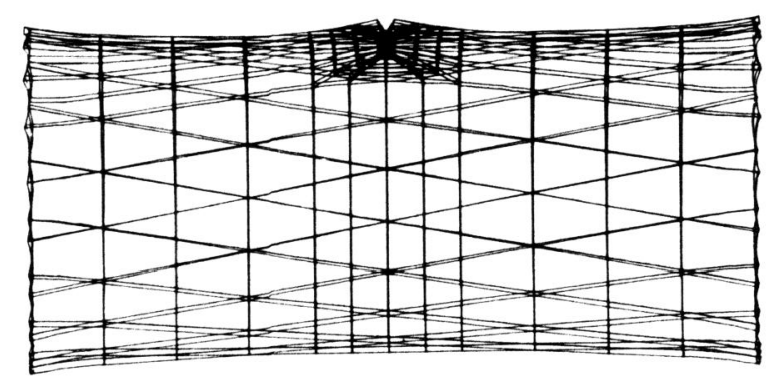

Fig.7. Finite element model of a cylinder with $\alpha=20^{\circ}$ and $\mathrm{R} / \mathrm{t}=25$ under combined tension and torsion.

stress intensity components can be determined for the individual cases of tension or torsion from the shallov-shell theory (or FEM). The resulting mixed mode loading, then, for combined tension and torsion can be determined with $K_{I}=f\left(G_{m}, G_{b}\right)$ and $K_{I I}=f\left(G_{m}, G_{b}\right)$ at the crack tip. For long cracks, however, the stress flntensiey factors have to

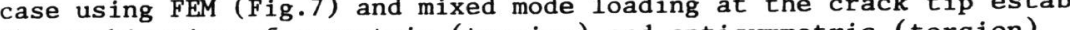

transverse around the crack.

\section{CONCLUSIONS}

1. Deformation of a cylindrical shell with a circumferential crack is highly dependent on the type of loading. Tensile loading produces crack opening displacements and a symmetric out-of-plane peformation rack. In torsion, the deformation and crack sliding displacements.

2. For very thin shells with long circumferential cracks under uniform membrane loading (tension or torsion) the shallow-shell theory is not malide validity of the theory is restricted to $\lambda_{2}<3$ for thin shells with $R / t=25$.

3. The combined loading (tension and torsion) produces different mixed mode loading conditions at the crack tip for different crack lengths.

\section{REFERENCES}

Delale, F. and Erdogan F. (1979). Transverse shear effect in a circumferentially cracked cylindrical she11, Quarterly of Applied Mathematics, 37, 239-258.

Duncan-Fama, M.E. and Sanders, Jr. J.L. (1972). A circumferential crack in a cylindrical shel1 under tension. Int. J. Fracture Mech. 8, 15-20.

Erdogn shells containing a circumferential crack, Int. J. Fracture. Mech. 6, 379-392.

Erdogan, F. and Ratwani, M. (1972). A circumferential crack

Fol shell under torsion, lnt. shell, Int. J. Fracture. Mech. 3, 1-11.

Kumosa, M. and Hul1, D. (1988a). Finite element analysis of a circumferentially cracked cylindrical shell under uniform tensile loading, to be published in Engineering Fracture Mechanics.

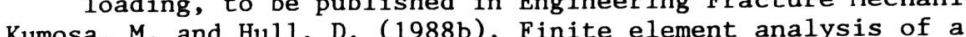
cir. and to be published in Engineering Fracture Mechanics.

Kurce cylindrical shell containing a circumferential crack, the proceedings cylindrical shell containing a circumferential crack, the proceedings March 1988, University of Liverpoo1.

Laksminarayana H.V. and Murthy M.V.V. (1976). On stress around an arbitrarily oriented crack in a cylindrical shell, Int. J. Fracture, $12,547-566$.

Murthy, M.V.V, Rao, K.P. and Rao, A.K. (1974). On the stress problem of large elliptical cutouts and cracks in circular cylindrical shells, Int. J. Solids Structures, $10,1243-1269$.

M. (1976). Crack extension modeling with singular quadratic isoparametric elements, Int. J. Fracture, 12 647-651. 